\title{
Geometric Characterization of a Singly Charged Oxygen Vacancy on a Single-Crystalline MgO(001) Film by Electron Paramagnetic Resonance Spectroscopy
}

\author{
Martin Sterrer, Esther Fischbach, Thomas Risse, ${ }^{*}$ and Hans-Joachim Freund \\ Department of Chemical Physics, Fritz-Haber-Institut der Max-Planck-Gesellschaft, Faradayweg 4-6, D-14195 Berlin, Germany
}

(Received 11 February 2005; published 12 May 2005)

\begin{abstract}
Electron paramagnetic resonance spectra of singly charged surface oxygen vacancies ( $F$ or color centers) formed by electron bombardment on a single-crystalline $\mathrm{MgO}$ film under UHV conditions are reported. The embedding of the defect in a well-defined geometrical environment allows not only for the determination of the magnetic quantities but also, in conjunction with STM studies, for the geometrical assignment of the observed signal to color centers located on the edges of the $\mathrm{MgO}$ film.
\end{abstract}

PACS numbers: 68.55.Ln, 68.37.Ef, 73.20.Hb, 76.30.Mi

It has long been recognized that the properties of metal oxide surfaces are strongly modified by the presence of defects. Specifically, point defects play an important role in this respect. In oxides an important class of point defects is oxygen vacancies, often called color centers ( $F$ centers) due their specific optical absorption, which has been known from bulk single crystals studies for a very long time [1]. The interest in the counterparts on the surface is largely triggered by the importance of these surfaces in catalysis. In particular, the possibility to donate or abstract electrons to adsorbed species, being molecules or small deposited metal particles, is considered an important mechanism to change the catalytic properties of such systems considerably [2]. Even though the importance of color centers on the surface properties is generally accepted, a microscopic experimental characterization of these centers in terms of their electronic and geometric structure and their influence on adsorbed species is still lacking. In this Letter, we present, for the first time, electron paramagnetic resonance (EPR) measurements under UHV conditions in conjunction with STM images of paramagnetic surface color centers on a single-crystalline $\mathrm{MgO}$ film, which allow not only a determination of the characteristic magnetic quantity but also a characterization of the geometric structure of the observed surface centers.

Divalent alkaline earth metal oxides, and, in particular, $\mathrm{MgO}$, are prototype systems to study color centers. Different anion vacancies are found in these systems, primarily characterized by the number of trapped electrons. Three different charge states of the vacancy, denoted $F^{2+}, F^{+}$, and $F^{0}$, are expected. The bulk $F^{0}$ and $F^{+}$centers have been studied in detail in single crystal samples by optical spectroscopy [3]. The latter species are paramagnetic and are thus susceptible to EPR spectroscopy, which has also been applied to characterize these centers. In the bulk of $\mathrm{MgO}$, the unpaired electron of the $F^{+}$center is embedded in the symmetric octahedral crystal field of the six surrounding $\mathrm{Mg}^{2+}$ neighbors giving rise to an isotropic EPR signal around the $g$ value of the free electron [4]. EPR measurements on $\mathrm{MgO}$ powders have shown that similar species are also present on the surface [5]. In contrast to the bulk, the surface centers were able to react with gas phase molecules such as oxygen. Furthermore, the EPR position of surface color centers becomes anisotropic with respect to the orientation of the static magnetic field due to the reduced symmetry of the defect. Formally, the Zeeman interaction is no longer characterized by an isotropic $g$ value, but it may be described by a symmetric $(3 \times 3)$ interaction matrix, called $g$ tensor, which can always be diagonalized in the molecular framework. The situation on a surface is complicated further by the fact that the color center can be located in different local environments. The most important configurations discussed in the literature so far are vacancies on flat terraces, on-line defects such as edges, and at corners [6]. The $g$ tensors for all situations should be different and, more importantly, they are unknown. Therefore, a correlation of the $g$ tensor with structural properties of the defect is not possible for a powder containing all possible orientations, as long as no specific interactions with a known geometric relationship can be probed. Since the latter situation is difficult to establish experimentally, the assignment needs support by theoretical modeling. This comparison is particularly challenging since the $g$-tensor anisotropy of color centers on $\mathrm{MgO}$ is in general small, which requires highly accurate calculations [7]. Because of these complications, isolated color centers have not been studied in any detail so far, despite the large body of work on $\mathrm{MgO}$ powders that has been performed in the last years to characterize a variety of paramagnetic centers, including color centers with an neighboring $\mathrm{OH}$ group, on $\mathrm{MgO}$ surfaces [8,9].

To correlate the EPR spectra with the geometric structure of the color center, we aim at orientation selection in the EPR spectra using color centers with a well-defined geometrical relationship to the laboratory framework. Therefore, surface color centers on a thin, epitaxially grown $\mathrm{MgO}(001)$ film on $\mathrm{Mo}(001)$ were investigated under UHV conditions by EPR spectroscopy. For the investigation of paramagnetic species on single crystal surfaces, absolute sensitivity is of paramount importance. It has 
been shown previously that EPR spectroscopy has the necessary sensitivity to detect paramagnetic species in submonolayer coverage under UHV conditions [10]. From these experiments a minimal sensitivity of $1 \times 10^{12}$ spins is deduced assuming a typical linewidth for color centers of $1.5 \mathrm{G}$.

The pristine $\mathrm{MgO}$ film exhibits no EPR signal above the noise floor of the apparatus; therefore the number of singly charged color centers is lower than $1 \times 10^{12}$ on this surface. This is in line with observations by the Pfnür group, who failed to see states in the band gap of pristine $\mathrm{MgO}$ films on $\operatorname{Ag}(001)$ [11]. Paramagnetic color centers can be produced in different ways. Here, the pristine film was exposed to electrons with an energy of $100 \mathrm{eV}$. The production of color centers in such a way was shown recently by specific losses observed in the electron energy loss spectrum of $\mathrm{MgO}$ films grown on $\mathrm{Ag}(001)[11,12]$. The defect generation by electron bombardment was explained by electron stimulated desorption of oxygen atoms or ions via a multielectron Auger decay resulting from excitation of $\mathrm{Mg} 2 s$ and $2 p$ electrons [11].

The morphology of $\mathrm{MgO}$ films grown epitaxially on $\operatorname{Ag}(001)$ and $\mathrm{Mo}(001)$ has been studied by scanning tunneling microscopy [13-15]. In Fig. 1 we report a lowtemperature (4 K) STM image of a $\mathrm{MgO}$ thin film with 4 monolayer nominal thickness deposited on $\operatorname{Ag}(001)$, which has been irradiated with electrons with an energy of
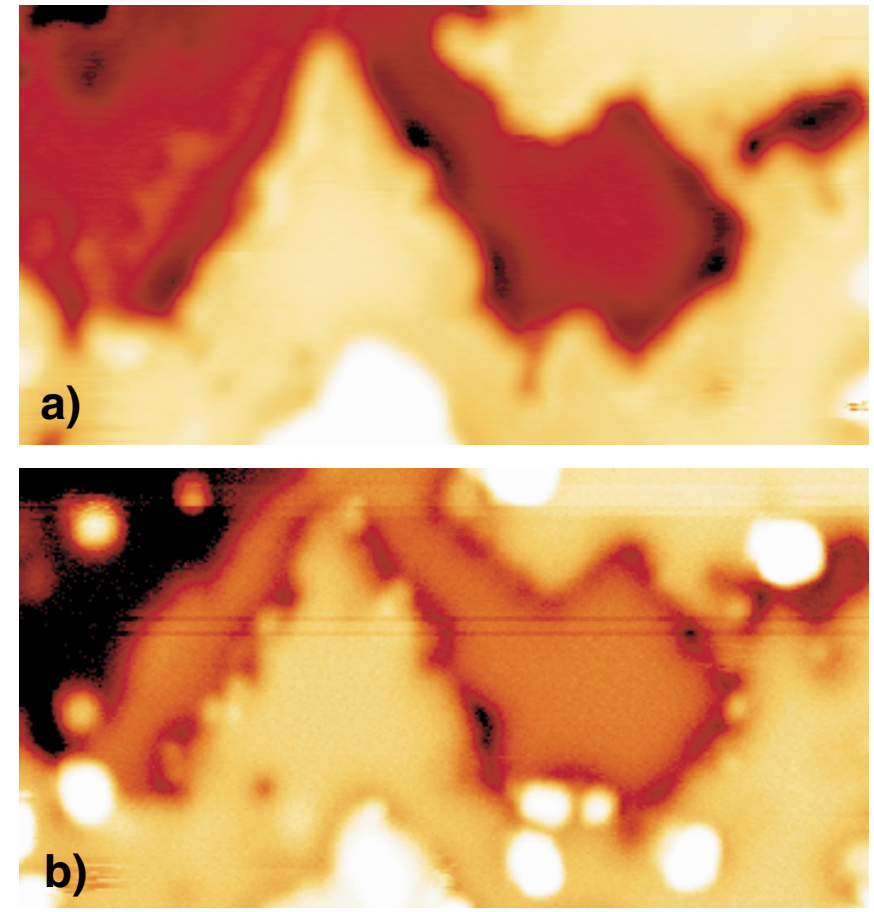

FIG. 1 (color). Low-temperature $(4 \mathrm{~K})$ STM images $(28 \times$ $16 \mathrm{~nm}^{2}$ ) of 4 monolayer $\mathrm{MgO}(001) / \mathrm{Ag}(001)$ after electron bombardment. (a) shows the morphology of the $\mathrm{MgO}$ film, measured at $U_{\text {bias }}=+3.5 \mathrm{~V}$. Enhanced contrast for defects is obtained for $U_{\text {bias }}=-2.5 \mathrm{~V}$ (b).
$100 \mathrm{eV}$. At high positive bias voltage [Fig. 1(a)], tunneling occurs via the unoccupied states of the $\mathrm{MgO}$ film and the morpholgy of the film is imaged. In accordance with previous data, we observe square-shaped $\mathrm{MgO}$ islands with edges running along the $\langle 100\rangle$ crystal directions of the film. Under special tunneling conditions, here $U_{\text {bias }}=$ $-2.5 \mathrm{~V}$, both the morphology and the electronic defects of $\mathrm{MgO}$ islands are imaged by STM [Fig. 1(b)]. Apart from the morphological features that are also observed in Fig. 1(a), spots of different size and brightness appear in Fig. 1(b). These are due to trapped electron centers $\left(F^{0}\right.$ and $F^{+}$) and are associated with point defects induced by electron bombardment [16]. From the STM image, it can clearly be seen that the majority of the defects are located at the edges and corners of the islands rather than on the regular (100) surface.

For an EPR spectroscopic description of the color center defects, the directions of the three principal components of the $g$ tensor $\left(g_{x}, g_{y}, g_{z}\right)$ have to be chosen such as to reflect the symmetry at the defect. As shown in Fig. 2 we have assumed ideal symmetry at the defect sites, which is $C_{4 v}$ on the terrace, $C_{2 v}$ on the edge, and $C_{3 v}$ at the corner. Correspondingly, one $g$-tensor component, which is usually chosen to be the $z$ or parallel component, is oriented

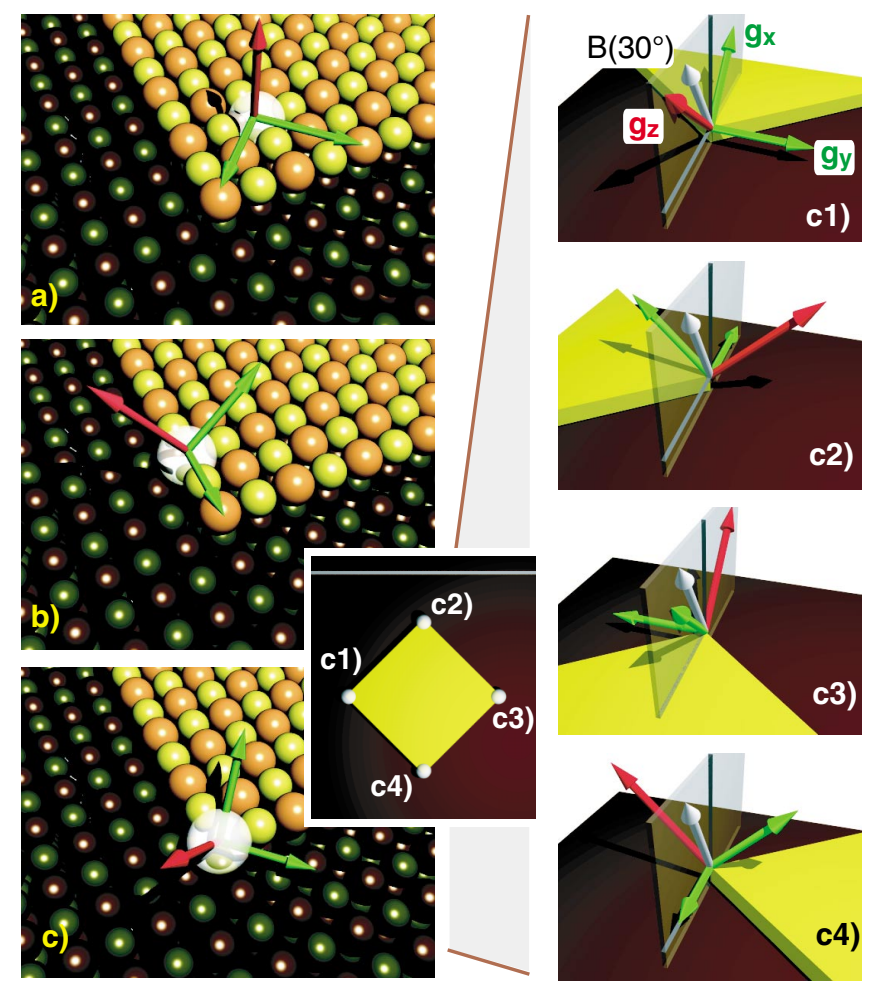

FIG. 2 (color). Left panels: Schematic representation of $g$-tensor components for single charged anion vacancies located on (a) terraces, (b) edges, and (c) corners of $\mathrm{MgO}(001)$ islands supported on $\mathrm{Mo}(001)$. Right panels: Orientation of the $g$ tensor with respect to the magnetic field for four symmetry related corner sits. 
along the high symmetry direction (axis of rotation) as seen in Fig. 2 (red arrows). For $C_{4 v}$ and $C_{3 v}$ symmetry, an axial tensor $\left(g_{\mathrm{par}}:=g_{z}, g_{x}=g_{y}:=g_{\mathrm{per}}\right)$ is expected, which renders the choice of the other directions in the orthogonal plane arbitrary. For $C 2_{v}$ symmetry (edges), all tensor components can be different and the principal axes are oriented as indicated in Fig. 2(b).

Prior to a discussion of the experimental results, it is instructive to examine the effect of a well-defined geometric relationship between surface color centers and the laboratory framework on the EPR spectrum. For a defect located on the surface terrace [Fig. 2(a)] of singlecrystalline $\mathrm{MgO}$, only a single configuration of the color center with respect to the laboratory framework is expected. However, in addition to the corner and edge sites indicated in Figs. 2(b) and 2(c), respectively, symmetry related sites exist due to the fourfold symmetry of the $\mathrm{MgO}$ islands. As an example, in Fig. 2 (c1-c4) all symmetry related corner sites, which are present on rectangularshaped $\mathrm{MgO}$ islands, are shown.

The implication of a variation of the direction of the magnetic field with respect to the surface on the angular dependence of the EPR spectra may be analyzed using the vector diagram shown for different corner site configurations in Fig. 2 (c1-c4). In the present experimental setup, the direction of the static magnetic field can be rotated in a plane spanned by the surface normal and the [001] direction of the $\mathrm{Mo}(001)$ single crystal surface as indicated in Fig. 2. The resonance position of the symmetry related paramagnetic defects at the given orientation of the magnetic field is determined by an effective $g$ value according to

$$
g(\theta, \varphi)=\left(\sin ^{2} \theta \cos ^{2} \varphi g_{x}^{2}+\sin ^{2} \theta \sin ^{2} \varphi g_{y}^{2}+\cos ^{2} \theta g_{z}^{2}\right),
$$

where $\theta$ is the angle between $B$ and $g_{z}$, and $\varphi$ is the corresponding polar angle in the $x y$ plane of the $g$ tensor. Thus the line shape of the EPR spectrum is given by the superposition of the resonances for the four sites. A careful analysis of the pictures $\mathrm{c} 1-\mathrm{c} 4$ reveals that, for the given orientation of $B\left(\theta=30^{\circ}\right)$, situations c 2 and $\mathrm{c} 4$ are equivalent, whereas $\mathrm{c} 1$ and $\mathrm{c} 3$ are mutually different from these. This statement holds true for all angles but $\theta=0^{\circ}$, where all situations are equivalent, and $\theta=90^{\circ}$, where c1 and c3 become degenerate. The effective $g$ value is the same for all corner sites for magnetic field alignment along the surface normal $\left(0^{\circ}\right)$. However, for both $30^{\circ}$ and $90^{\circ}$, various contributions of the parallel and perpendicular components occur for the different defect configurations [Fig. 2 (c1-c4)] yielding a superposition of two resonances at $90^{\circ}$ and three resonances at $30^{\circ}$. Taking into account the small $g$-tensor anisotropy of surface color centers on $\mathrm{MgO}$ and a finite linewidth of the signals, the EPR spectra at different magnetic field angles are expected to differ only by small variations in linewidth and resonance position. Terrace and edge sites may be analyzed correspondingly.
The experimental ESR spectra of color centers generated by electron bombardment on the surface of a 20 monolayer $\mathrm{MgO}$ film are shown in Fig. 3. The surface nature of these centers was proven by their complete disappearance after reaction with $1 \mathrm{~L}\left(10^{-6}\right.$ torr s) of molecular oxygen. For the magnetic field aligned parallel $\left(0^{\circ}\right)$ and perpendicular $\left(90^{\circ}\right)$ with respect to the surface normal, the line shape is almost symmetric with similar linewidths of $1.3 \mathrm{G}$. However, the apparent resonance position of the parallel configuration is shifted by $0.5 \mathrm{G}$ to lower field with respect to the $90^{\circ}$ spectrum. In contrast, a spectrum taken at a polar angle of $30^{\circ}$ is significantly broader and its line shape is no longer symmetric. The resonance field position is only slightly different to the $0^{\circ}$ situation. This small difference in resonance positions already points at a small $g$-tensor anisotropy, which is generally observed for color centers on $\mathrm{MgO}[9,17]$. To extract the principal components of the $g$ tensor, one needs to resort to spectral simulations. As already discussed above, the results of the simulation depend on the assumptions made for the nature of the color center. For the current set of data the best fit was achieved by assuming the color centers to be located at edge sites. Figure 3 shows these fits superimposed on the experimental spectra. The corresponding $g$-tensor parameters are given in Table I. It is interesting to note that the tensor is nearly axially symmetric within our experimental uncertainties, although the symmetry of the site would allow for an orthorhombic tensor. This indicates that the in plane anisotropy of the tensor imposed by the crystal field is relatively small in this case. For the spectra taken at $0^{\circ}$ and $90^{\circ}$, the simulation of all three locations produce equally good fits as judged by the reduced- $\chi^{2}$ values. However, the fits to the spectrum taken

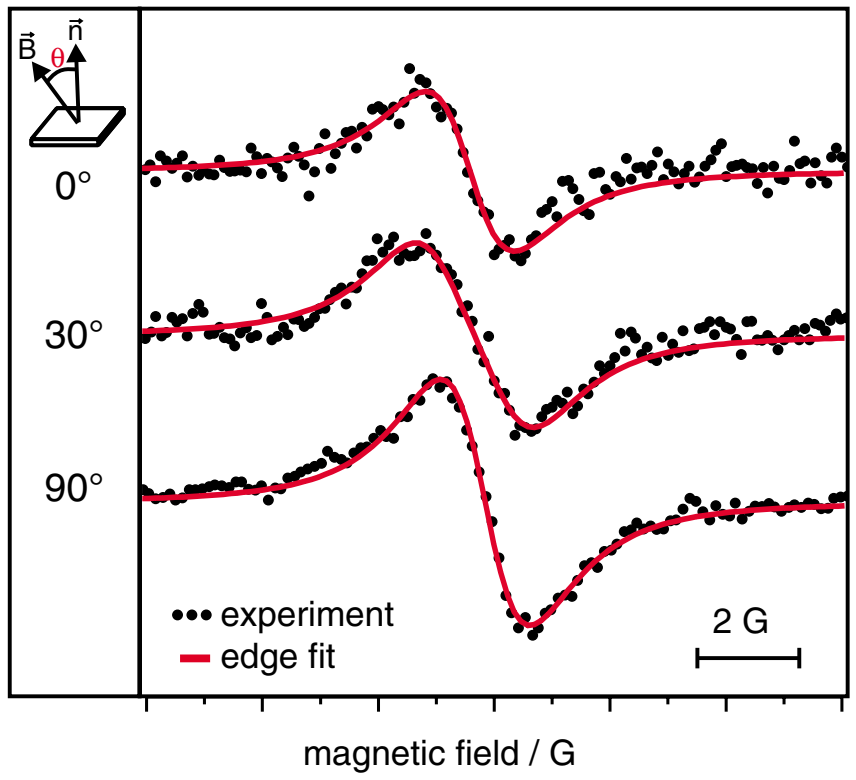

FIG. 3 (color). Experimental and simulated EPR spectra of color centers on 20 monolayer $\mathrm{MgO}(001) / \mathrm{Mo}(001)$. 
TABLE I. $g$ parameters of color centers located on edges and corners of $\mathrm{MgO}$ islands $\left[g_{\text {iso }}=\frac{1}{3}\left(g_{x}+g_{y}+g_{z}\right), \quad \Delta g=\right.$ $\left.g_{z}-g_{\text {iso }}, \eta=\left(g_{y}-g_{x}\right) / \Delta g\right]$.

\begin{tabular}{lccc}
\hline \hline Site & $g_{\text {iso }}$ & $\Delta g$ & $\eta$ \\
Edge & $2.0001 \pm 0.00005$ & 0.00040 & 0.25 \\
Corner & $2.0001 \pm 0.00005$ & 0.00027 & 0 \\
\hline \hline
\end{tabular}

at $30^{\circ}$ are different for the three situations, which is documented by the reduced- $\chi^{2}$ values given in Table II. As seen from these values the best fit is produced assuming edges, followed by corner sites (for $g$-tensor parameters, see Table I). The terrace sites give the worst fit, which is due to the fact that the terrace situation will always be characterized by a single configuration and thus a symmetric line.

On the basis of fits for the edge sites, it cannot be excluded that a certain fraction of corner sites is simultaneously present. From fits of both components the fraction of the corner sites can be estimated to be below $20 \%$. This correlates well with the STM data presented above and is in line with the picture that color centers located at lowcoordinated sites are more stable than those at highercoordinated sites [6].

In conclusion, we have presented, for the first time, EPR spectra of color centers on single-crystalline substrates under UHV conditions that allow an identification of the geometric property of the paramagnetic defect in addition to the accurate determination of the principal components of an almost isotropic $g$ tensor. The paramagnetic color centers generated by electron bombardment on the surface of $\mathrm{MgO}$ thin films are located predominantly at the edges of the $\mathrm{MgO}$ facets, a result deduced from the angular dependence of the line shape.

Experimental procedures. -EPR experiments were carried out in an UHV chamber equipped with standard UHV parts for thin film preparation and characterization which has been described in detail elsewhere [18]. The Mo(001) substrate was cleaned by oxidation at $1500 \mathrm{~K}$ in $1 \times$ $10^{-6}$ mbar $\mathrm{O}_{2}$ and subsequent annealing to $2300 \mathrm{~K}$. A 20 monolayer thin $\mathrm{MgO}$ film was prepared by evaporation of $\mathrm{Mg}$ at $10^{-6}$ mbar oxygen background pressure and a substrate temperature of $600 \mathrm{~K}$ [19]. The formation of a crystalline $\mathrm{MgO}(100)$ film was verified using LEED. Color centers were generated by electron bombardment from a tantalum filament at electron doses of 100-300 electrons per surface oxygen ion and an energy of $100 \mathrm{eV}$. ESR measurements were performed at room temperature at a microwave frequency of $9.67 \mathrm{GHz}$ ( $X$ band) and a microwave power of $2 \mathrm{~mW}$. Prolonged data accumulation
TABLE II. Reduced- $\chi^{2}$ values of fits to the spectrum at $\theta=$ $30^{\circ}$ and the total reduced $\chi^{2}$ for all three spectra using different models. In addition, the optimal linewidth for each model is given.

\begin{tabular}{lccc}
\hline \hline Site & Linewidth & Reduced $\chi^{2}\left(30^{\circ}\right)$ & Reduced $\chi^{2}$ (total) \\
Edge & $1.10 \mathrm{G}$ & 1.033 & 1.052 \\
Corner & $1.06 \mathrm{G}$ & 1.122 & 1.071 \\
Terrace & $1.13 \mathrm{G}$ & 1.21 & 1.085 \\
\hline \hline
\end{tabular}

typically over $12 \mathrm{~h}$ was necessary to achieve the present signal-to-noise ratio. DPPH (2,2-Diphenyl-1picrylhydracil radical) was used for $g$-value calibration $(g=2.0036)$. For low-temperature STM measurements, a 4 monolayer thin $\mathrm{MgO}$ film was grown on $\mathrm{Ag}(001)$. The substrate was cleaned by repeated $\mathrm{Ar}^{+}$sputtering and subsequent annealing to $700 \mathrm{~K}$. MgO film deposition and electron bombardment were performed as described above.

We thank Hans-Peter Rust, Markus Heyde, and Marek Nowicki (FHI-Berlin) for support with the lowtemperature STM experiments. M.S. is grateful for financial support by the Austrian Science Fund (FWF), ErwinSchrödinger Grant No. J2345-B10. E.F. thanks the Studienstiftung des deutschen Volkes for financial support.

*Electronic address: risse@ fhi-berlin.mpg.de

[1] B. Henderson and J. E. Wertz, Adv. Phys. 17, 749 (1968).

[2] B. Yoon et al., Science 307, 403 (2005).

[3] Y. Chen, R. T. Williams, and W. Sibley, Phys. Rev. 182, 960 (1969).

[4] J.E. Wertz et al., Phys. Rev. 107, 1535 (1957).

[5] R. L. Nelson and A. J. Tench, J. Chem. Phys. 40, 2736 (1964).

[6] G. Pacchioni and P. Pescarmona, Surf. Sci. 412-413, 657 (1998).

[7] K. M. Neyman et al., J. Phys. Chem. A 106, 5022 (2002).

[8] M. Chiesa et al., Angew. Chem., Int. Ed. 42, 1759 (2003).

[9] M. Sterrer et al., Chem. Phys. Chem. 5, 1695 (2004).

[10] H. Schlienz et al., Phys. Rev. Lett. 74, 761 (1995).

[11] J. Kramer et al., Surf. Sci. 517, 87 (2002).

[12] J. Kramer, C. Tegenkamp, and H. Pfnuer, Phys. Rev. B 67, 235401 (2003).

[13] S. Schinkte et al., Phys. Rev. Lett. 87, 276801 (2001).

[14] M. C. Gallagher et al., Thin Solid Films 445, 90 (2003).

[15] S. Valeri et al., Phys. Rev. B 65, 245410 (2002).

[16] M. Sterrer et al. (to be published).

[17] E. Giamello et al., J. Phys. Chem. B 101, 971 (1997).

[18] J. Schmidt et al., J. Chem. Phys. 116, 10861 (2002).

[19] M.-C. Wu et al., J. Vac. Sci. Technol. A 10, 1467 (1992). 